\title{
Model of Handover and Traffic Based on Cellular Geometry with Smart Antenna
}

\author{
Zufan Zhang, ${ }^{1}$ Jie Zhang, ${ }^{1}$ and Shaohui Sun ${ }^{2}$ \\ ${ }^{1}$ Chongqing Key Labs of Mobile Communications Technology, Chongqing University of Posts and Telecommunications, \\ Chongqing 400065, China \\ ${ }^{2}$ Datang Wireless Mobile Innovation Center, China Academy of Telecommunication Technology (CATT), Beijing 100191, China \\ Correspondence should be addressed to Zufan Zhang; zhangzf@cqupt.edu.cn
}

Received 17 February 2014; Accepted 5 April 2014; Published 17 April 2014

Academic Editor: Xiaoli Chu

Copyright (C) 2014 Zufan Zhang et al. This is an open access article distributed under the Creative Commons Attribution License, which permits unrestricted use, distribution, and reproduction in any medium, provided the original work is properly cited.

Based on the application of smart antennas in cellular mobile communications, this paper introduces the impact of the width of the antenna beams playing on the dwell time probability density function in cellular geometry with smart antenna. The research results indicate that the smart cell structure can improve the dwell time of users within the cell and improve the traffic system performance.

\section{Introduction}

The handover is key feature and unique feature of the cellular mobile communication system [1-3]. The handover means the process of a call from one cell to another cell when the user moves in the cellular coverage area. In order to meet the rapid growth of the demand of the wireless communication, we need to improve the system capacity radically. The means to improve the system capacity which are related to the structure of cell are reducing the size of cell, adopting cell splitting technology, using concentric technology, or the hierarchical cellular structure. However, these means which can improve the system performance by changing the structures will make the management of handover more complicated. Thus, plenty of controlling strategies and algorithms emerged.

In order to analyze these controlling strategies and algorithms, the performance indicators such as call hold time and dwell time can be regarded as the measurements [4]. Call hold time and dwell time depend on the user mobility, location, and handover controlling strategies. In order to properly indicate the call hold time and dwell time, the better mobility model is necessary. Simply, we assume that the cell has special shapes such as hexagon or round. When this kind of model combines with the distribution of specific speed and the moving direction, it can determine the probability density function of dwell time. But, in reality, the shapes of cells are irregular and the velocity and direction of the mobile user may be difficult to characterize. Thus it will be more appropriate to regard the dwell time as the random variable of the probability density function. So we can obtain full information about the cell shapes and user mobility. Zonoozi and Dassanayake used the general gamma distribution, Orlik and Rappaport used ultraexponential distribution, and Fan and Chlamtac used hyper-Erlang distribution to characterize the probability density function of dwell time.

The characterization of the call hold time and dwell time described above plays an important role in analyzing the improved handover performance. We assumed that the locations of users within a specific study area are distributed uniformly [5], and we suppose that the shape of cell is round. And we are less likely to analyze the sector cell. We can combine the user location probability density function with cellular geometry to analyze the impacts played by the call dwell time and hold time. Furthermore, we propose the impact of the width of the antenna beams in smart cell playing on the dwell time probability density function, so we further study the relationship among the users dwell time, traffic, and structure of smart cell with smart antennas. 


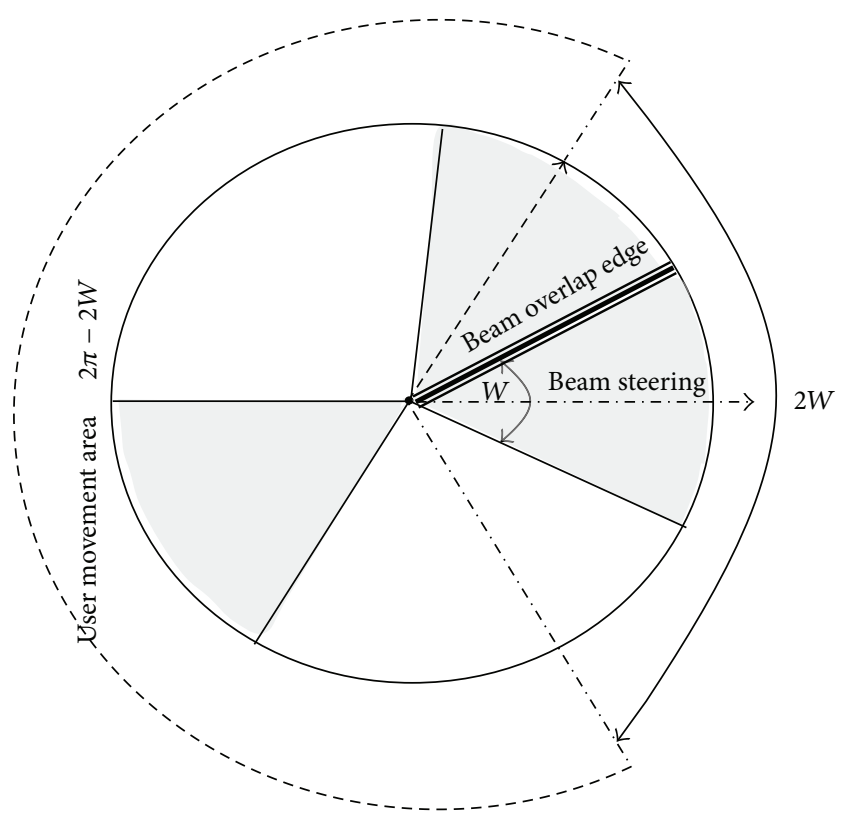

FIGURE 1: User movement area within the cellular geometry with smart antenna.

\section{Characteristics of Users Movements with Smart Antenna}

One significant feature of the cell with smart antennas is that the frequency can be reused many times within the same cell [6]. Part of the base stations within this kind of cell can dynamically track mobile users [7], so it can increase the dwell time of user movement. We assume that the same frequency is used two times within a cell, shown in Figure 1, and the width of antenna beam is $W$-the minimum resolvable angle between two users-namely, when the angle between two users who share the same channel is less than $W$, it has to handover. We usually call this progress intracell handover [5-8]. Considering that two users who share the same frequency may be near the border of two adjacent antenna beams and the distance between the two users has to be $W$, shown in Figure 1, the maximal moving area of a user within cell is a sector whose area is $2 \pi-2 W$, and we ignore the intercell handover.

The relative moving speed has to be considered in smart antenna cell. We assume that the two velocities of two mobile stations $\mathrm{MS}_{1}, \mathrm{MS}_{2}$ share the same frequency but in the different antenna beams are $\vec{V}_{1}\left(V_{1}, \alpha_{1}\right)$ and $\vec{V}_{2}\left(V_{2}, \alpha_{2}\right)$, where $V_{1}, V_{2}$ are the random variable speeds of movements of $\mathrm{MS}_{1}$ and $\mathrm{MS}_{2}$ and $\alpha_{1}, \alpha_{2}$ are the random variable of two moving directions. We suppose that $V_{1}, V_{2}$ have the same probability density of function $f_{V_{i}}\left(V_{i}\right)(i=1,2)$ and $\alpha_{1}, \alpha_{2}$ have the same probability density function $f_{a_{i}}\left(a_{i}\right)(i=1,2)$. Thus, the moving speed $\vec{V}_{r}\left(V_{r}, \alpha_{r}\right)$ of $\mathrm{MS}_{2}$ which is respected to $\mathrm{MS}_{1}$ can be described as

$$
\vec{V}_{r}\left(V_{r}, \alpha_{r}\right)=\vec{V}_{2}\left(V_{2}, \alpha_{2}\right)-\vec{V}_{1}\left(V_{1}, \alpha_{1}\right)
$$

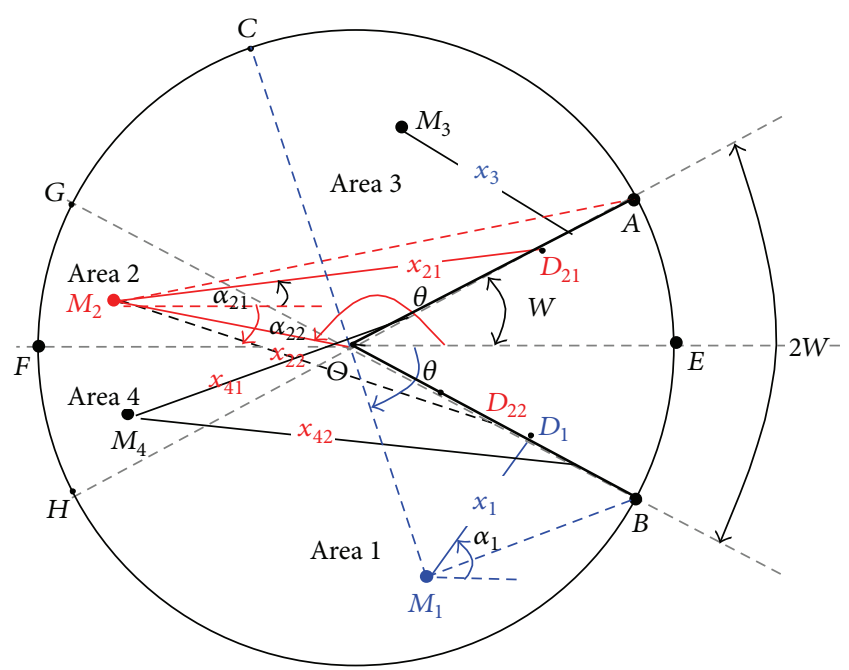

FIGURE 2: Diagram of user dwell time.

where $V_{r}$ could be described as follows:

$$
\begin{aligned}
& V_{r}=\sqrt{V_{1}^{2}+V_{2}^{2}-2 V_{1} V_{2} \cos \left(\alpha_{1}-\alpha_{2}\right)}, \\
& \underline{\underline{\alpha=\alpha_{1}-\alpha_{2}}} \sqrt{V_{1}^{2}+V_{2}^{2}-2 V_{1} V_{2} \cos \alpha}, \\
& f_{V_{1}, V_{2}, \alpha}\left(V_{1}, V_{2}, \alpha\right)=f_{V_{1}}\left(V_{1}\right) \cdot f_{V_{2}}\left(V_{2}\right) \cdot f_{\alpha}(\alpha) \text {, } \\
& f_{\alpha}(\alpha)=f_{\alpha_{1}}\left(\alpha_{1}\right)=f_{\alpha_{2}}\left(\alpha_{2}\right) \\
& V_{r}=V_{r} \\
& u=V_{1} \text {, } \\
& v=V_{2}, \\
& f_{V_{r}, u, v}\left(V_{r}, u, v\right)=|J| f(u, v, \alpha), \\
& |J|=\left|\frac{\partial \alpha}{\partial V_{r}}\right|=\frac{2 V_{r}}{\sqrt{\left(2 V_{1} V_{2}\right)^{2}-\left(V_{1}^{2}+V_{2}^{2}-V_{r}^{2}\right)^{2}}}, \\
& f_{V_{r}}\left(V_{r}\right)=\iint_{-\infty}^{\infty} f_{V_{r}, u, v}\left(V_{r}, u, v\right) d u d v
\end{aligned}
$$

\section{Probability Density Function of Dwell Time with Smart Antenna}

We create the schematic diagram about the user dwell time shown in Figure 2. We can divide the movement areas into 4 parts. It is obvious that areas 1 and 3 are symmetrical and areas 
2 and 4 are symmetrical. We can get the following formula by geometric analysis.

In area $1, x_{1}$ is

$$
x_{1}=\frac{r \sin (\theta-W)}{\sin \left(\alpha_{1}+W\right)},
$$

where $\theta$ and $\alpha_{1}$ sacrifice the following formula:

$$
\begin{gathered}
W \leq \theta \leq \pi-W \\
\sin ^{-1} \frac{r \sin (\theta-W)}{\sqrt{r^{2}+R^{2}-2 R r \cos (\theta-W)}}-W \leq \alpha_{1} \leq \pi-\theta .
\end{gathered}
$$

In area $2, x_{21}$ is

$$
x_{21}=\frac{r \sin (\theta-W)}{\sin \left(W-\alpha_{21}\right)},
$$

where $\theta$ and $\alpha_{21}$ sacrifice the following formula:

$$
\begin{gathered}
\pi-W \leq \theta \leq \pi \\
\theta-\pi \leq \alpha_{21} \leq W-\sin ^{-1} \frac{r \sin (\theta-W)}{\sqrt{r^{2}+R^{2}-2 R r \cos \theta}} .
\end{gathered}
$$

In area $2, x_{22}$ is

$$
x_{22}=\frac{r \sin (2 \pi-\theta-W)}{\sin \left(W-\alpha_{22}\right)},
$$

where $\theta$ and $\alpha_{22}$ sacrifice the following formula:

$$
\begin{gathered}
\pi-W \leq \theta \leq \pi \\
\pi-\theta \leq \alpha_{22} \leq W-\sin ^{-1} \frac{r \sin (\theta-W)}{\sqrt{R^{2}+r^{2}-2 R r \cos \theta}} .
\end{gathered}
$$

We can get that the conditional probability density functions $f_{x_{i}}^{a n}(x \mid \theta)$ of $x_{1}, x_{21}$, and $x_{22}$ are

$$
f_{x_{i}}^{a n}(x \mid \theta)=\int_{-\infty}^{\infty}\left|J_{i}^{n}\right| f_{r, i}^{n}\left(u, \alpha_{i}\right) d u, \quad(i=1,21,22),
$$

where

$$
\begin{gathered}
\left|J_{1}^{n}\right|=\left|\frac{\partial \alpha_{1}}{\partial x_{1}}\right|=\frac{r \sin (\theta-W)}{x_{1} \sqrt{x_{1}^{2}-r^{2} \sin ^{2}(\theta-W)}}, \\
\left|J_{21}^{n}\right|=\left|\frac{\partial \alpha_{21}}{\partial x_{21}}\right|=\frac{r \sin (\theta-W)}{x_{21} \sqrt{x_{21}^{2}-r^{2} \sin ^{2}(\theta-W)}}, \\
\left|J_{22}^{n}\right|=\left|\frac{\partial \alpha_{22}}{\partial x_{22}}\right|=\frac{r \sin (2 \pi-\theta-W)}{x_{22} \sqrt{x_{22}^{2}-r^{2} \sin ^{2}(\theta+W)}} .
\end{gathered}
$$

Thus, the probability density functions $f_{x_{i}}^{a n}(x \mid \theta)$ of $x_{1}$, $x_{21}$, and $x_{22}$ are

$$
f_{x_{i}}^{a n}(x)=\int_{\theta_{i}^{\prime}}^{\theta_{i}} f_{x_{i}}^{n}(x \mid \theta) f_{\theta_{i}}\left(\theta_{i}\right) d \theta, \quad(i=1,21,22),
$$

where

$$
\begin{aligned}
& \theta_{1}^{\prime}=W, \quad \theta_{1}=\pi-W, \\
& \theta_{21}^{\prime}=\pi-W, \quad \theta_{22}=\pi, \\
& \theta_{22}^{\prime}=\pi-W, \quad \theta_{22}=\pi .
\end{aligned}
$$

By symmetry, we can further get that the probability density functions of $x_{3}, x_{41}$, and $x_{42}$ are

$$
f_{X_{3}}^{a n}(x)=f_{X_{1}}^{a n}(x), f_{X_{41}}^{a n}(x)=f_{X_{21}}^{a n}(x), f_{X_{42}}^{a n}(x)=f_{X_{22}}^{a n}(x) .
$$

If $X_{i}=T_{i} V(i=1,21,22,3,41,42)$, then

$$
f_{T_{i}}^{a n}(t)= \begin{cases}\int_{V_{\min }}^{V_{\max }} v f_{X_{i}}^{a n}(t v) f_{v}^{a n}(v) d v, & 0<t \leq \frac{R}{V_{\max }} \\ \int_{V_{\min }}^{R / t_{i}} v f_{X_{i}}^{a n}(t v) f_{v}^{a n}(v) d v, & t>\frac{R}{V_{\max }} .\end{cases}
$$
user is

Thus the probability density distribution of the calling

$$
F_{T}^{a n}(t)=P\{T \leq t\}
$$

$$
\begin{gathered}
=\iint_{0}^{\infty} P\left(T_{1} \leq t \mid T_{21}=\tau_{21}, T_{22}=\tau_{22},\right. \\
\left.T_{3}=\tau_{3}, T_{41}=\tau_{41}, T_{42}=\tau_{42}\right) \\
\times d F_{T_{21}}^{a n}\left(\tau_{21}\right) d F_{T_{22}}^{a n}\left(\tau_{22}\right) d F_{T_{3}}^{a n}\left(\tau_{3}\right) \\
\times d F_{T_{41}}^{a n}\left(\tau_{41}\right) d F_{T_{42}}^{a n}\left(\tau_{42}\right) .
\end{gathered}
$$
user is

And the probability density distribution of the handover

$$
\begin{gathered}
F_{T}^{a h}(t)=P\{T \leq t\} \\
=\iint_{0}^{\infty} P\left(T_{1} \leq t \mid T_{21}=\tau_{21}, T_{3}=\tau_{3},\right. \\
\left.T_{42}=\tau_{42}\right) d F_{T_{21}}^{a h}\left(\tau_{21}\right) \\
\times d F_{T_{3}}^{a h}\left(\tau_{3}\right) d F_{T_{42}}^{a h}\left(\tau_{42}\right) .
\end{gathered}
$$

Generally, considering that the smart antenna cell has the ability to balance the traffic within the cell and intercell and can solve the problems of traffic "hot spots" effectively [8], 


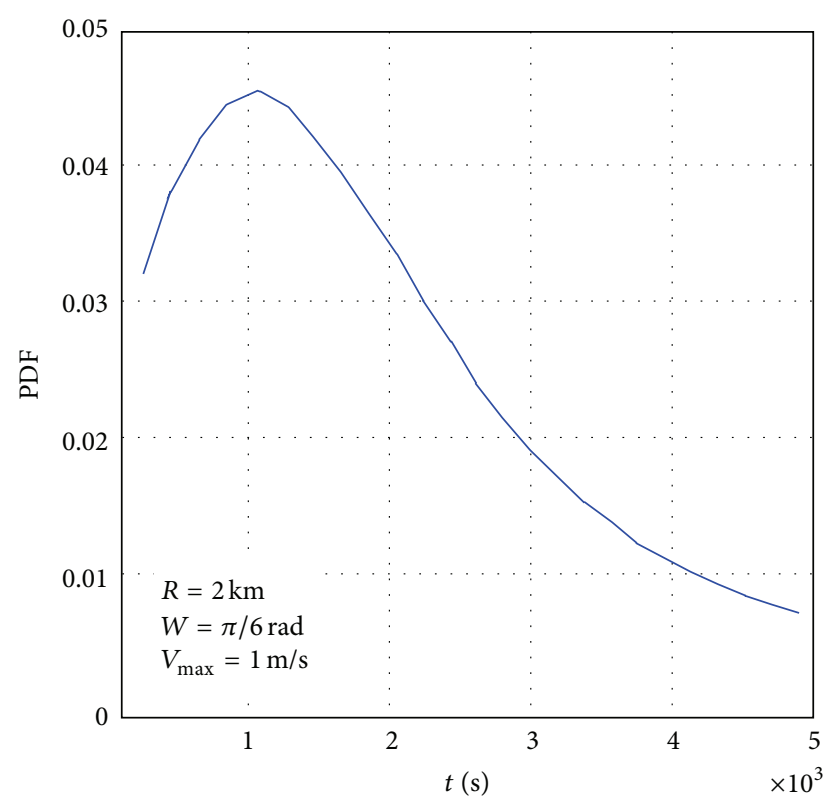

(a)

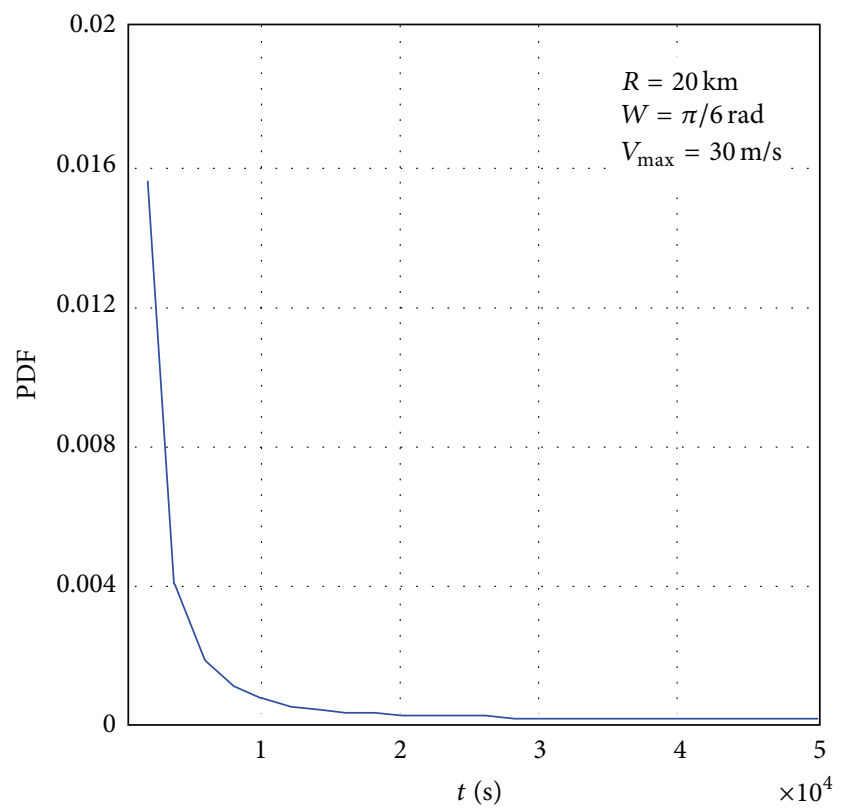

(c)

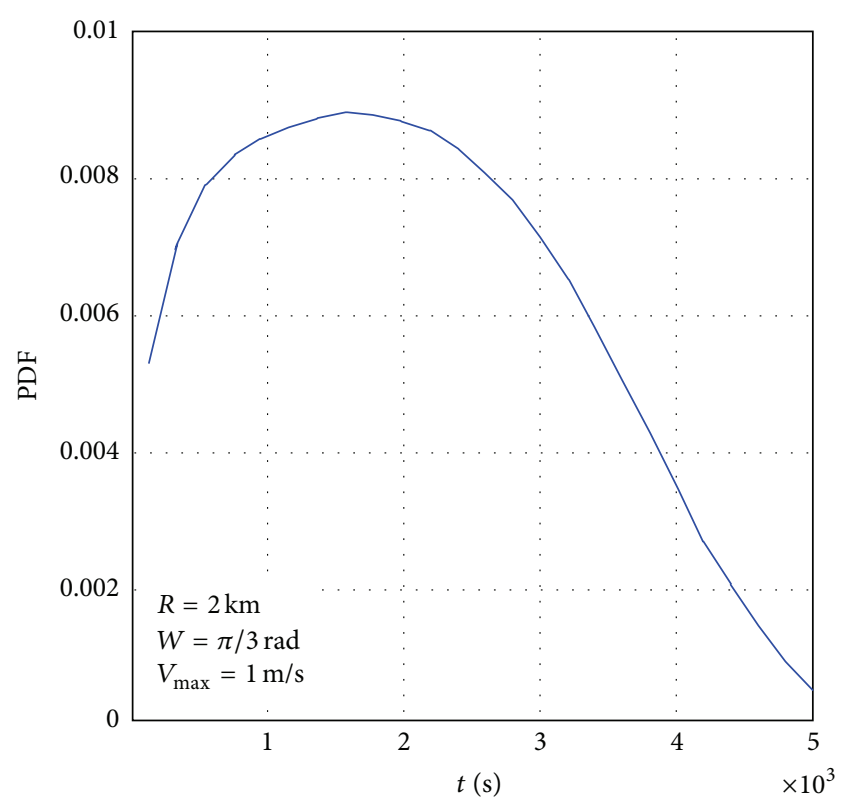

(b)

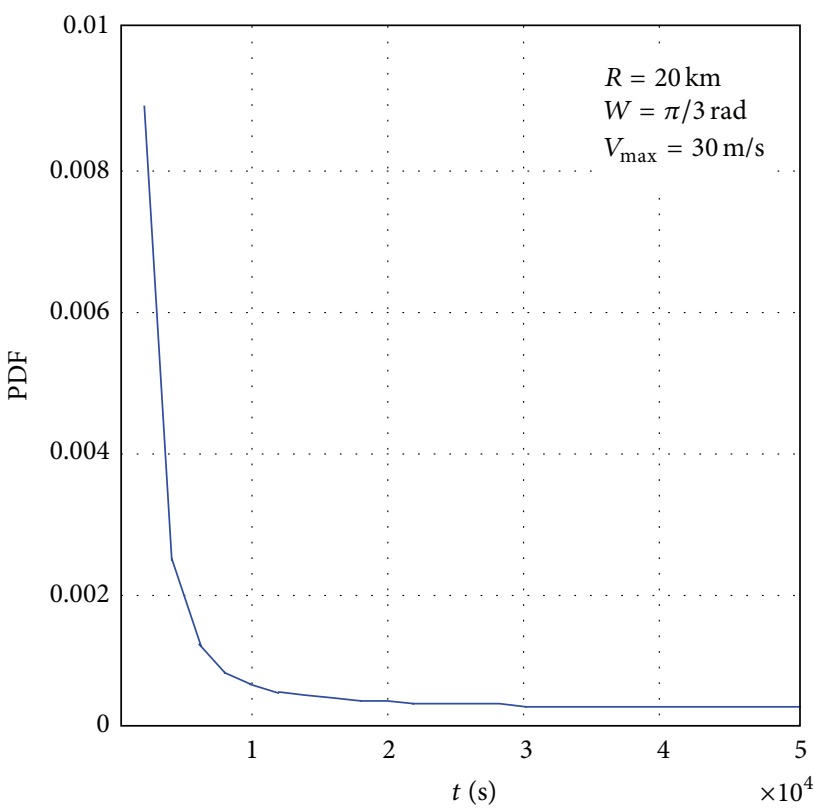

(d)

FIGURE 3: Calling user dwell time probability density function within the smart antenna cell.

we can assume that the users within the cell and the handover users who enter the cell from border are uniformly distributed.

The dwell time probability density function of smart antenna cell has been analyzed in Figures 3 and 4. The parameters are used to simulate the probability density function of the dwell time. It can be seen from Figures 3 and 4 that the performance of smart antenna cell is better than sector cell because this kind of cell can provide a longer movement distance.

\section{Traffic Analysis with Smart Antenna Cell}

As described above that the smart antennas have been used in the base station, $N$ channels can be used simultaneously within the cell. We suppose that the channels are to be reused only once here. When the number of calling users is smaller than or equal to $N$, the calling users can access the system; namely, the probability of accessing is 1 . When the number of calling users is larger than or equal to $N$ but smaller than or equal to $2 \mathrm{~N}$, considering that we have assumed that there 


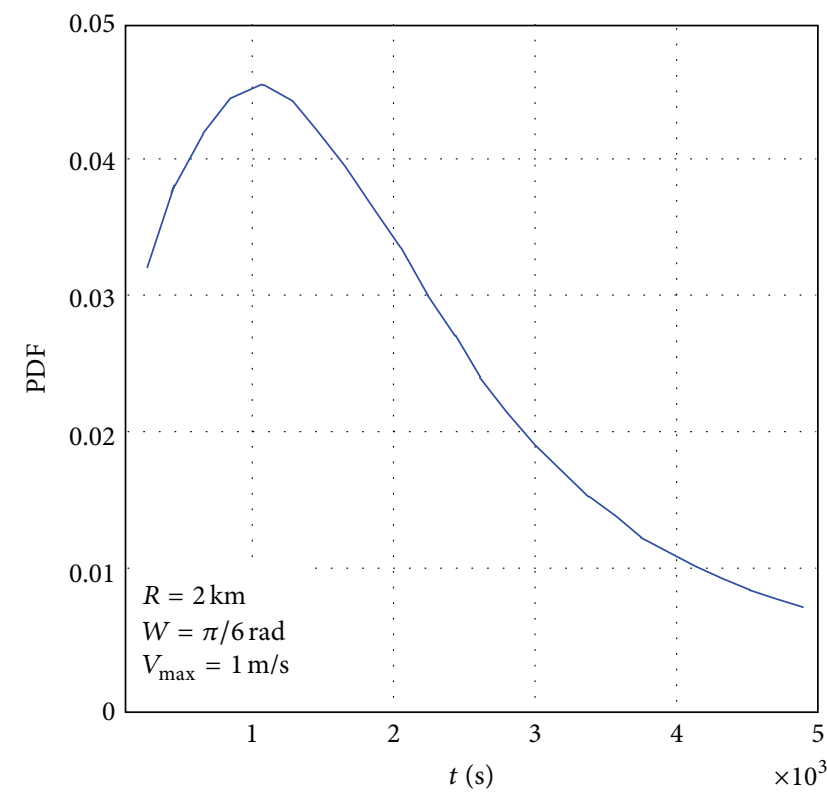

(a)

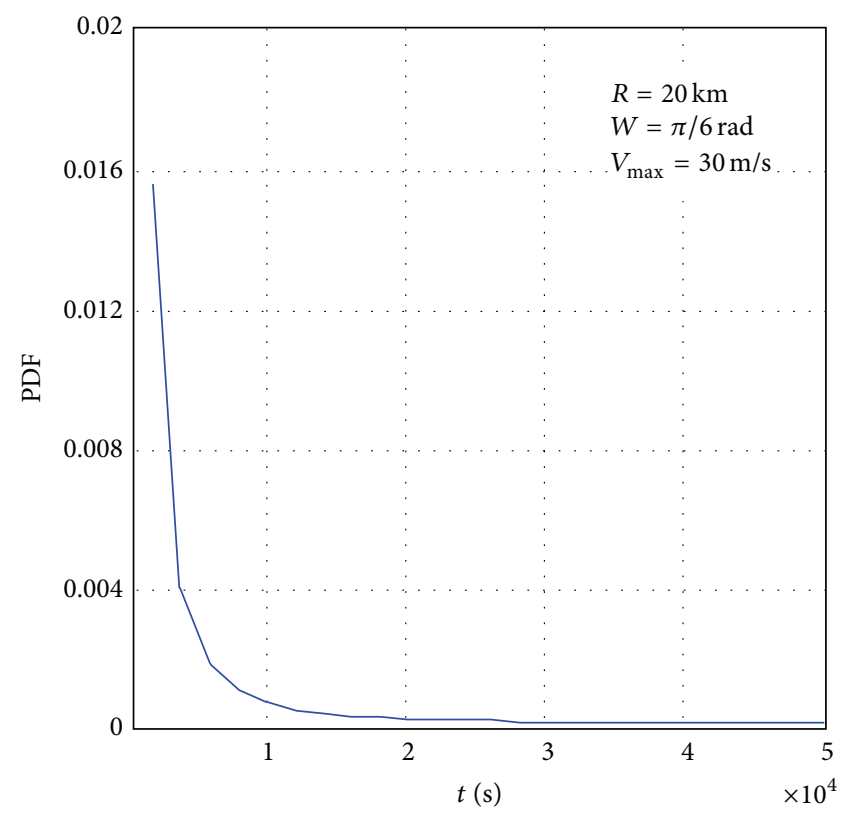

(c)

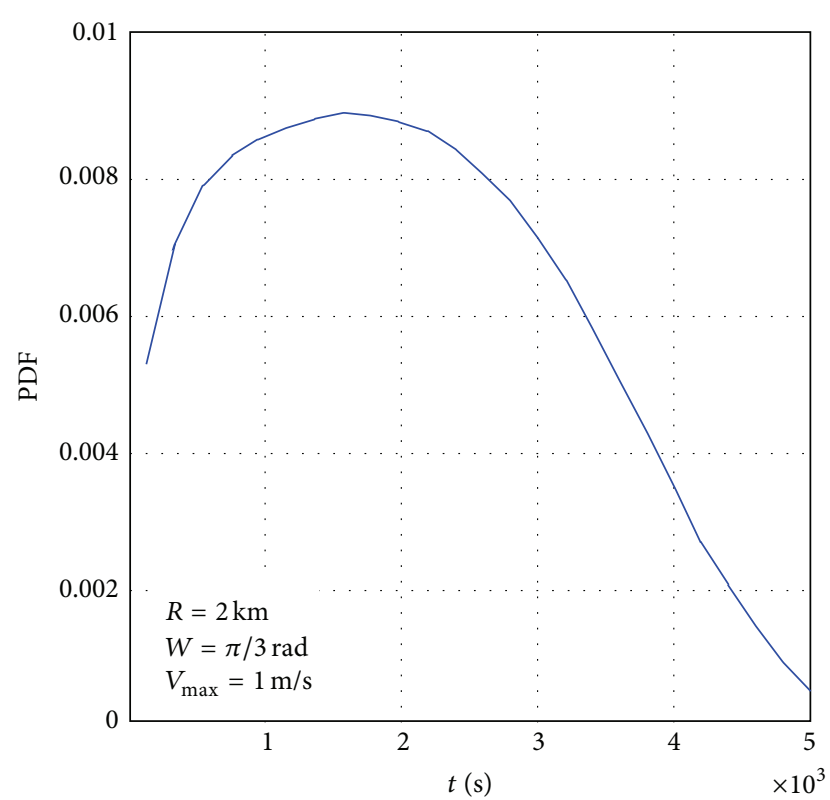

(b)

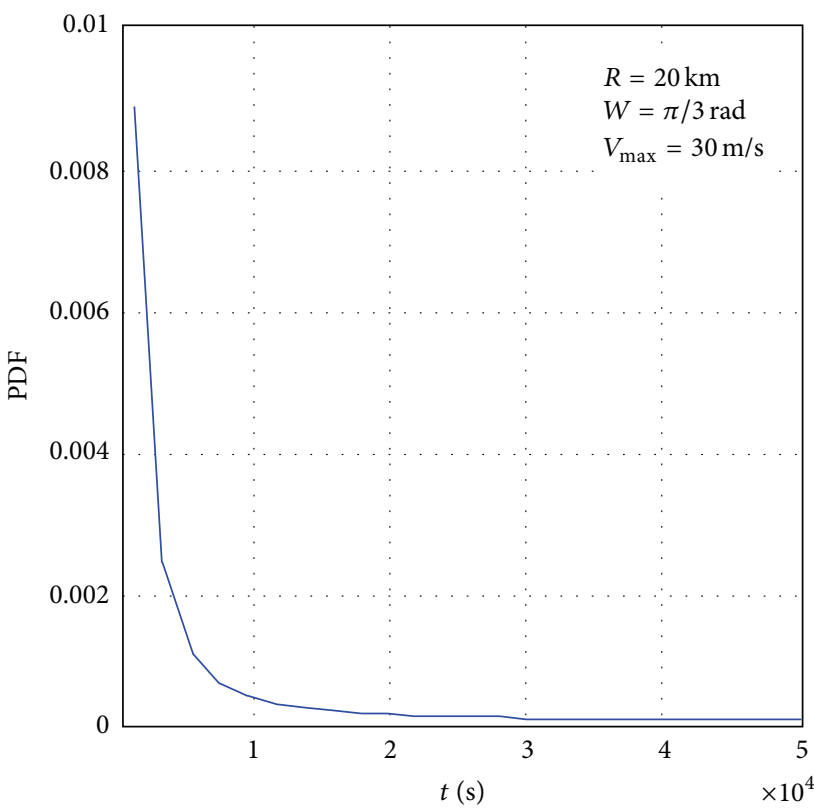

(d)

Figure 4: Handover user dwell time probability density function within the smart antenna cell.

are $2 \mathrm{~N}$ available channels as well as the beam overlap, the blocking probability is $(W / \pi)^{2 N-n}$. Thus, the probability of successful access to the system within cell is $p_{s}(n)$ [9]:

$$
p_{s}(n)= \begin{cases}1, & 0 \leq n<N \\ 1-\left(\frac{W}{\pi}\right)^{2 N-n}, & N \leq n \leq 2 N .\end{cases}
$$

When the number of calling users within cell is smaller than $N$, the probability of successful handover is 1 . Meanwhile, when the number of new calling users is larger than or equal to $N$ but smaller than or equal to $2 N$, the blocking probability is $(W / 2 \pi)^{2 N-n}$ and the probability of unsuccessful handover within the cell is $p_{r}(n)$ :

$$
p_{r}(n)= \begin{cases}0, & 0 \leq n<N \\ \left(\frac{W}{2 \pi}\right)^{2 N-n}, & N \leq n \leq 2 N .\end{cases}
$$

We define:

$\lambda$ : the arrival rate of calling users within cell, 


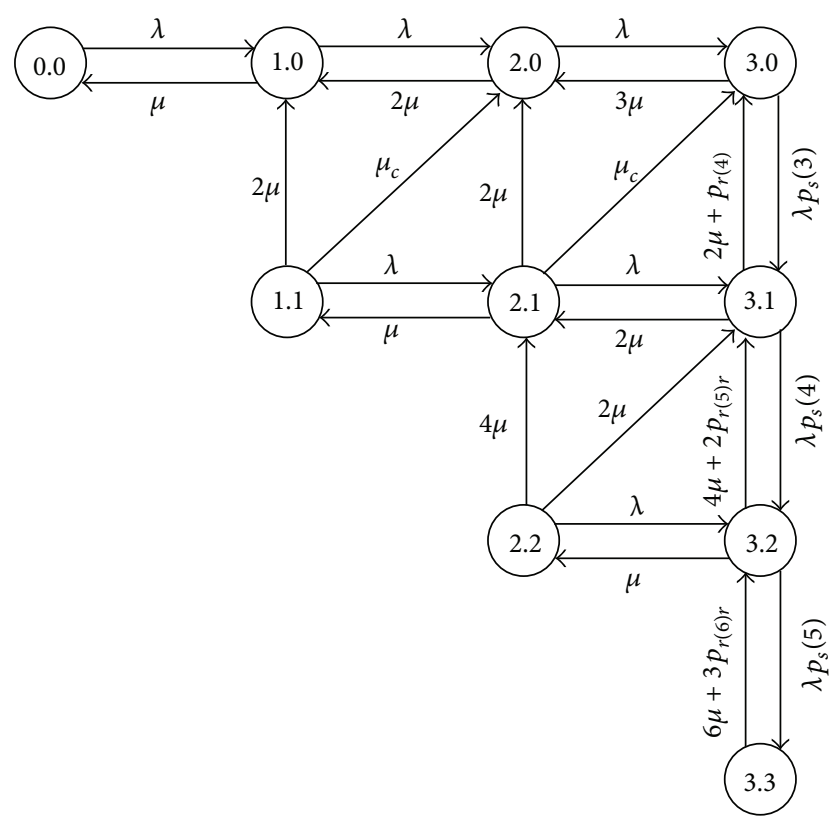

FIGURE 5: Channel state transition diagram with smart antenna cell.

$\mu$ : the leave rate of calling users who do not choose to handover within cell,

$\mu_{c}$ : the average leave rate of handover users within cell,

where

$$
\mu_{c}=\frac{1}{\int_{0}^{\infty}\left(1-F_{T}^{a n}(t)\right)}+\frac{1}{\int_{0}^{\infty}\left(1-F_{T}^{a h}(t)\right)} .
$$

We can get the state transition diagram shown in Figure 5. We can get the following balance equations from the above state transition diagram.

Consider the following: when $0 \leq j \leq i \leq N-1$

$$
\begin{aligned}
(i+j) & \mu p_{i, j}+\left(\lambda+j \mu_{c}\right) p_{i, j} \\
= & \lambda p_{i-1, j}+(i+1-j) \mu p_{i+1, j} \\
& +\mu_{c}(j+1) p_{i-1, j+1}+2 \mu(j+1)_{i, j+1},
\end{aligned}
$$

when $0 \leq j \leq i, i=N$,

$$
\begin{aligned}
p(N, j) & {\left[(j+N) \mu+j \mu_{c} p_{r}(N+j)+\lambda p_{s}(N+j)\right] } \\
= & p_{N, j+1}\left[2(j+1) \mu+(j+1) \mu_{c} p_{r}(j+1+N)\right] \\
& +\lambda p(N-1, j)+\lambda p(N, j-1) p_{s}(j-1+N) \\
& +\mu_{c} p(j+1)(N-1, j+1),
\end{aligned}
$$

and when $0 \leq i \leq j$

$$
\begin{gathered}
p_{i, j}=0, \\
\sum_{i=0}^{N} \sum_{j=0}^{N} p_{i, j}=1 .
\end{gathered}
$$

Thus, we can get that the blocking probability of calling users is $p_{n}$ :

$$
p_{n}=\sum_{j=0}^{N-1} p(N, j)\left[1-p_{s}(j+N)\right]+p(N, N)
$$

$$
\text { And the blocking probability of handover is } p_{h} \text { : }
$$

$$
p_{h}=\frac{\sum_{j=1}^{N} j \mu_{c} p_{N, j} p_{r}(j+N)}{\sum_{i=1}^{N} \sum_{j=1}^{N} j \mu_{c} p_{i, j}}=\frac{\sum_{j=1}^{N} j p_{N, j} p_{r}(j+N)}{\sum_{i=1}^{N} \sum_{j=1}^{N} j p_{i, j}} .
$$

A set of simulation results of relationship between $\lambda$ and blocking probabilities are shown in Figure 6. In order to compare with the above sector cell, the parameters are $1 / \mu_{0}=100(s)$ and $N=7$. We can know that (1) comparing with sector cell in [10], the blocking probabilities of calling users and handover users decline obviously; (2) when the speed is faster and the width of beam is wider, the blocking probabilities of new calling users and handover users are becoming closer. It is mainly because the wider beam reduces the effect of smart cell.

\section{Conclusions}

Combining with the present applications of smart antennas in cell, this paper mainly studies how to reduce the interference and how to enhance system capacity and so on. We introduce the impact of the width of the antenna beams playing on the dwell time probability density function in cellular geometry with smart antenna. Furthermore, we study how the structure of smart cell with smart antenna can influence the call dwell time and traffic within cell. We next will focus on the study of model of handover and traffic based on the mixed heterogeneous network geometry with smart antenna.

\section{Conflict of Interests}

The authors declare that there is no conflict of interests regarding the publication of this paper. 

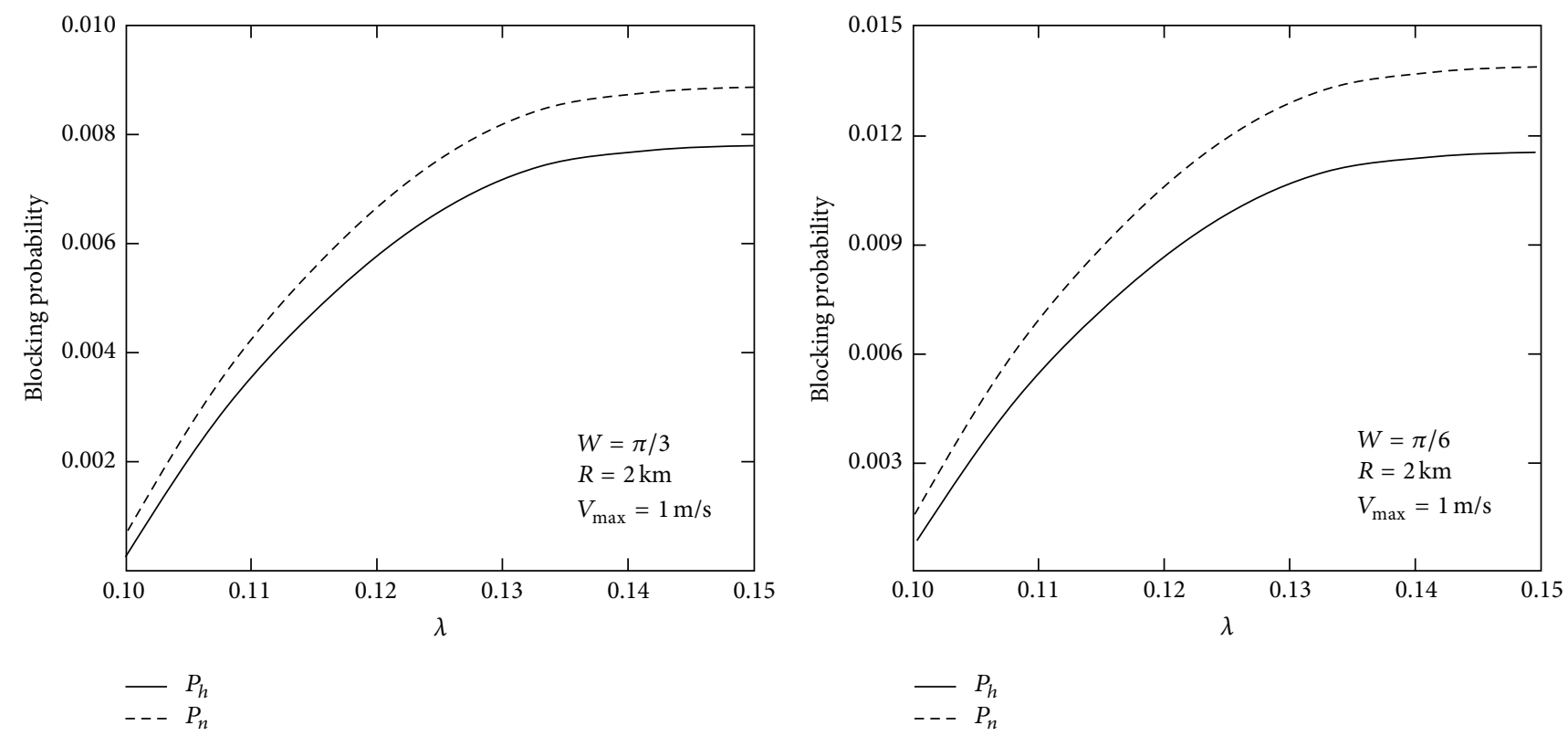

(a)
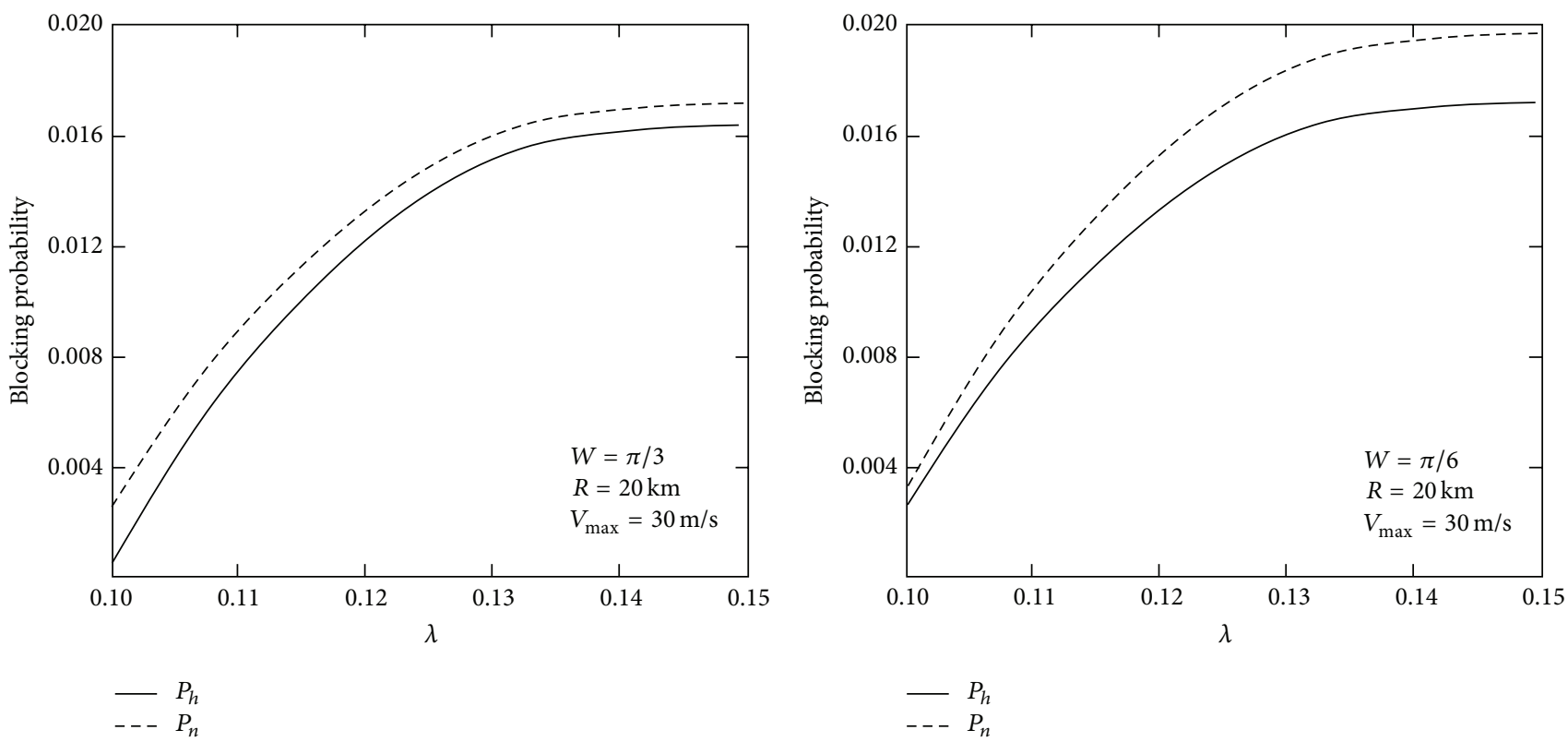

(c)

(d)

FIGURE 6: Relationship between $\lambda$ and blocking probabilities.

\section{Acknowledgments}

This work was jointly supported by the Chongqing Municipal Natural Science Foundation under Grant no. CSTC2013jjB40001, the National Key Scientific Instrument/ Equipment Development Project of China under Grant no. 2012YQ20022404, the National High Technology Research and Development Program of China (863 program) under Grant no. 2014AA01A705, and the Program for Changjiang Scholars and Innovative Research Team in University under Grant no. IRT1299.

\section{References}

[1] P. P. Bhattacharya, A. Sarkar, I. Sarkar, and S. Chatterjee, "An ANN based call handoff management scheme for mobile cellular network," International Journal of Wireless \& Mobile Networks, vol. 5, no. 6, pp. 125-135, 2013.

[2] M. Constantinescu, E. Borcoci, T. Rasheed, and D. Hayes, "An analysis of smart antenna usage for WiMAX vehicular communications," in Proceedings of the 3rd International ICST Conference (MOBILIGHT '12), pp. 248-257, October 2012.

[3] M. Constantinescu, "Optimization method for smart antenna use in WiMAX vehicular communications," in Proceedings of 
the 9th International Conference on Communications (COMM '12), pp. 263-266, June 2012.

[4] L. Ortigoza-Guerrero and A. Hamid Aghvami, "A prioritized handoff dynamic channel allocation strategy for PCS," IEEE Transactions on Vehicular Technology, vol. 48, no. 4, pp. 12031215, 1999.

[5] Z. Xu, A. H. N. Akansu, and S. Tekinay, "Cochannel interference computation and asymptotic performance analysis in TDMA/FDMA systems with interference adaptive dynamic channel allocation," IEEE Transactions on Vehicular Technology, vol. 49, no. 3, pp. 711-723, 2000.

[6] W.-J. Huang and J. F. Doherty, "An evaluation of blocking probability for three-fold SDMA," in Proceedings of the Communications for Network-Centric (Milcom '01), pp. 1248-1252, October 2001.

[7] Z. Rong, "Beamforming loss due to tracking error in downlink SDMA," in Proceedings of the IEEE International Conference on Universal Personal Communications (ICUPC '98), vol. of the 1998, pp. 441-444, October 1998.

[8] H. Liu, Y. Xu, and Q.-A. Zeng, "Modeling and performance analysis of future generation multimedia wireless and mobile networks using smart antennas," in Proceedings of the IEEE Wireless Communications and Networking Conference (WCNC '05), pp. 1286-1291, March 2005.

[9] Y. Kim, H. Ko, S. Pack, W. Lee, and X. Shen, "Mobility-aware call admission control algorithm with handoff queue in mobile hotspots," IEEE Transactions on Vehicular Technology, vol. 62, no. 8, pp. 3903-3411, 2013.

[10] Z. Zhang and H. Du, "Handover initiation analysis of a cell architecture in mobile communications systems," in Proceedings of the International Conference on Telecommunications (ICT '02), pp. 711-715, June 2002. 



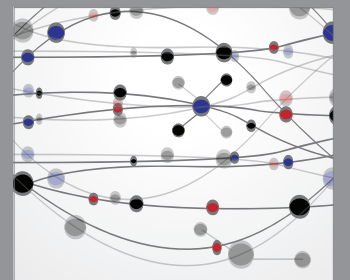

The Scientific World Journal
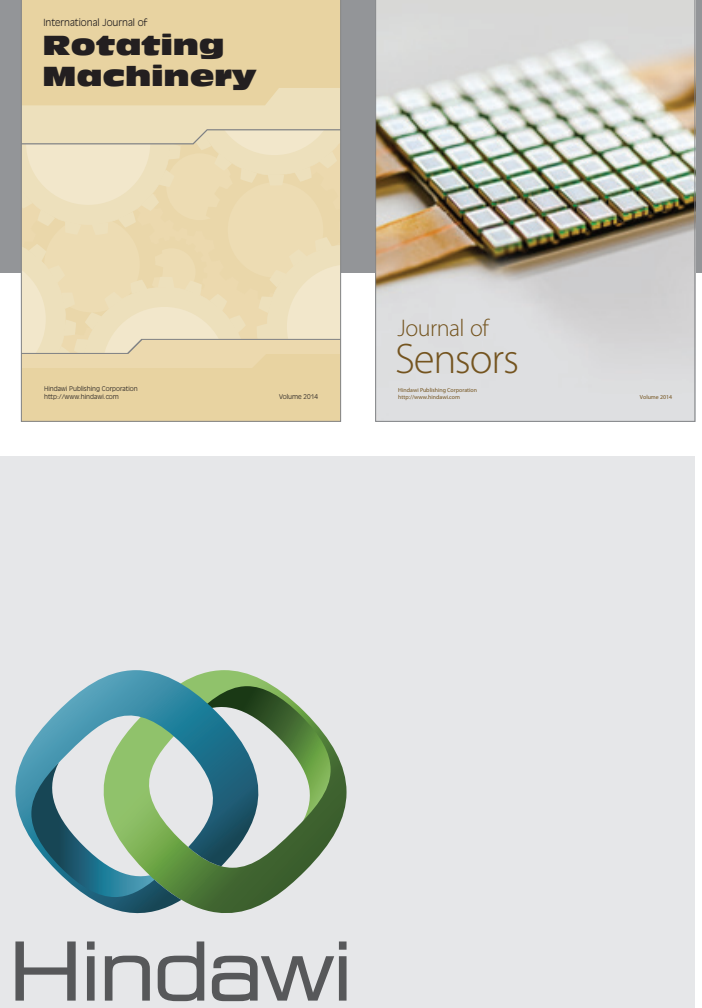

Submit your manuscripts at http://www.hindawi.com
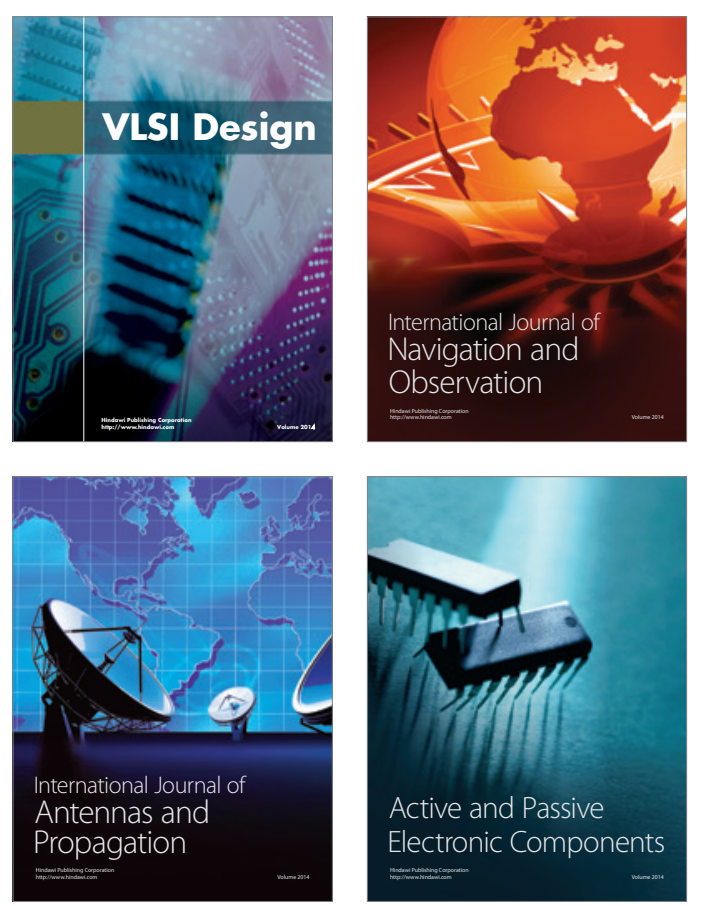
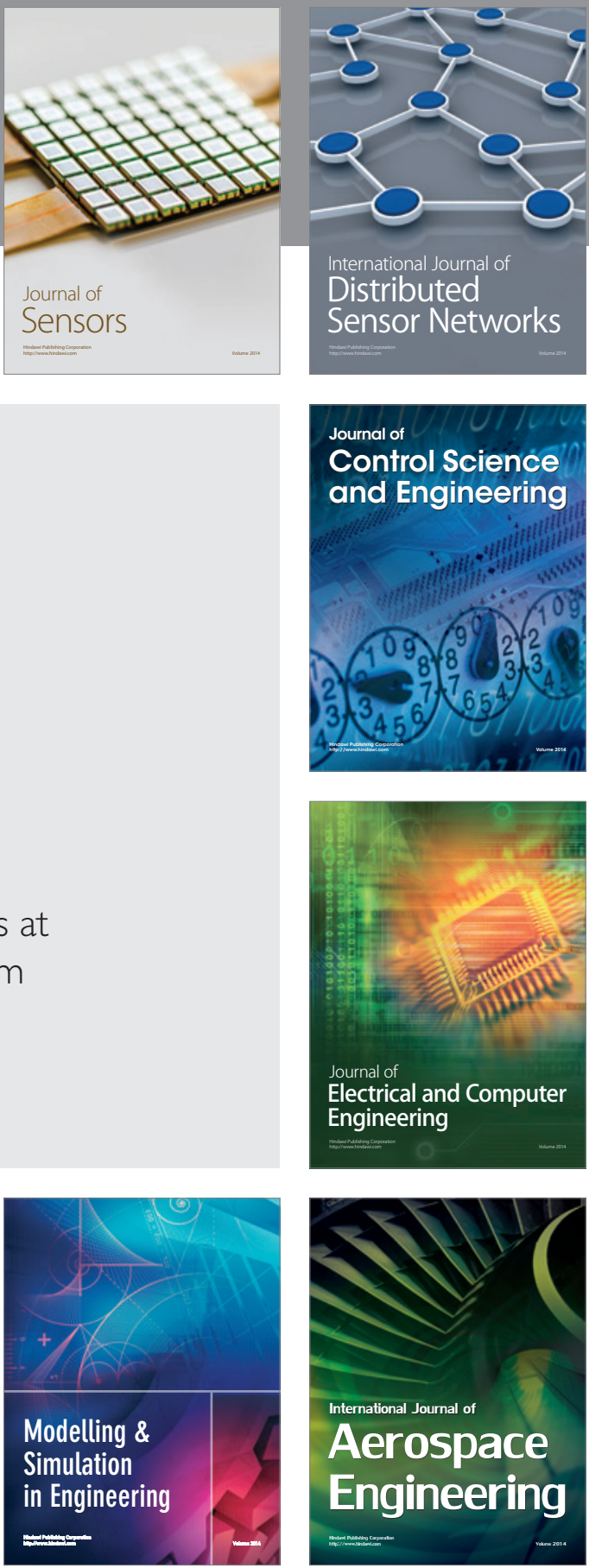

Journal of

Control Science

and Engineering
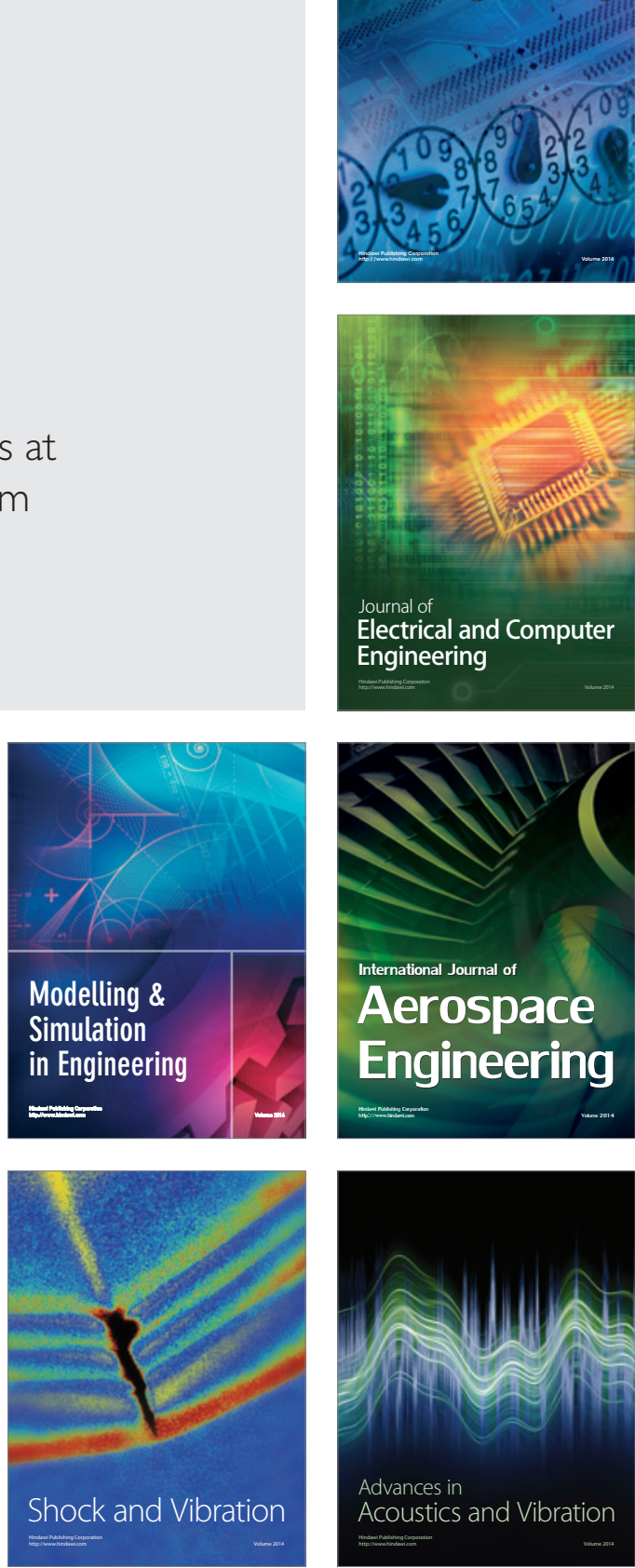